517. Phyllachora Salvadoræ Cooke. - Massana, ad folia Salvadore persica.

548. Podaxon arabicus Pat.

Scheikh Otman, Lahadj, wadi Mo'âden.

549. - Deflersii Pat. - Schoukra.

550. - pistillaris $\boldsymbol{F r}$. - Khamfer (Bilad Yafa).

551. Polyporus dichrous Pers. - In wadi Mo'âden, ad truncos

Fici morifolice.

552. Lentinus arabicus Pat. - Serrya.

553. Montagnites Haussknechtii Rab.

- Schoukra.

55 . Bolbitius arenarius Pat. Schoukra.

555. Coprinus hemerobius Fries. schoukra.

\title{
RECHERCHES SUR LA DIVISION DU NOYAU CELlULAIRE CHEZ LES
} VégétauX ( $8^{\circ}$ Note) (2); par M. Charles DEGAGNY.

Ici, toute description serait insuffisante. Pour donner une idée même approchée de ce qui arrive, il faut voir les préparations; alors les faits constatés sont nets, précis, saisissants. Lorsque les demi-bâtonnets se pelotonnent en formant le noyau fille, il n'est pas possible de ne pas être frappé de ce ramollissement, de cette diffluence de la linine qui forme un tout homogène. Puis, rapidement l'aspect change, la linine gonflée, ramollie, digérée, se condense, se contracte. Les contours, devenus invisibles, des demibâtonnets réapparaissent, confusément d'abord. Le filament est reconstitué; nous verrons comment.

Quand la linine s'est condensée, la nucléine avait déjà commencé à le faire. Quand la condensation des demi-bâtonnets devient apparente, c'est que la nucléine a cessé de diffuser à travers la linine, et de là entre les demi-plaques.

Et, en effet, en revenant aux fils du fuseau, il va être possible de voir à quel moment ils ont commencé à ne plus être digérés avec la mème intensité.

La digestion du fuseau n'est apparente, visible, constatable, que sur les fils de sa région. Entre les deux demi-plaques, quand les demi-bâtonnets se sont ramollis au point de former des coins qui s'aplatissent entre les fils, tout ce qui se trouve entre les demi-plaques, surtout les parties centrales soumises à l'in-

(1) Nomina a cl. Patouilla Rd benigne communicata.

(2) Voyez le Bulletin, plus haut, p. 310. 
fluence des deux, est dans un tel état de ramollissement qu'il n'est pas possible de ne pas se rendre à l'évidence. Or le fuseau tout entier, tout ce qu'il renferme de matières protoplasmiques subit la même influence; tout ce qui est fuseau est digéré. Mais tout ce qui respire à la périphérie, comme les couches voisines du protoplasma cellulaire, produit instantanément de l'énergie, reconstitue les molécules dédoublées par hydratation, condense des molécules à poids moléculaire élevé, en perdant de l'eau et de l'acide carbonique; et les fils se reforment. Les effets des hydratations sont supprimés aussitòt qu'ils se produisent. Ils le sont moins vite un peu plus profondément; et au centre ils persistent, et on les voit. Ramenés à un état plus jeune, imbibés des corps oxydables, réducteurs, diffusés par la nucléine, en respirant, les fils font emploi des forces vives produites; ils subissent une rénovation bien visible, et redeviennent ce qu'ils étaient à leur début. Mais, en revenant ce qu'ils étaient, ils répètent, une à une, la série des réaclions par lesquelles ils avaient passé antérieurement. De sorte qu'il n'est plus possible de douter de ces réactions et de leur point de départ; nous en aurons une confirmation nouvelle dans un instant.

Les fils extérieurs du fuseau qui respirent comme les parties voisines du cytoplasma produisent donc de l'énergie pour pouvoir se contracter au point de se rompre; et en continuant à respirer, à mesure qu'ils entrent dans le cytoplasma, ils produisent l'énergie suffisante non seulement pour se contracter, mais pour se raidir, pour produire des effets de tétanisation bien manifeste.

Les fils cassés augmentent en nombre, continuellement et très vite; ils divergent tous autour du fuseau, et forment deux espèces de parachutes qui s'ouvrent dans le cytoplasma, en même temps que les pointes du fuseau se rapprochent et que celui-ci se raccourcit; de sorte qu'il se produit aux pointes du fuseau une résistance qui contre-balance l'attraction produite sur elles par les fils qui, en se contractant dans le fuseau, tirent sur elles en même temps que les demi-bâtonnets en voie de progression.

Contigus aux fils extérieurs qui sont en train de casser, il existe une zone intermédiaire de fils qui s'incurvent en dehors en formant tonneau. Ces fils se contractent et tirent sur les pointes du fuseau. Il est facile de voir que c'est dans cette zone, et au fur et à mesure qu'ils deviennent plus superficiels, que les fils, en respi- 
rant de plus en plus, se contractent davantage et finissent par casser. On voit des fils qui viennent de se rompre, et d'autres dont la rupture est moins récente. Ces derniers continuent à se contracter, et leurs extrémités, tout à l'heure en contact, se sont éloignées l'une de l'autre. Autour du fuseau on voit les fils qui se sont rompus les premiers, ils sont beaucoup plus courts; il n'y a pas à s'y tromper, et le spectacle est vraiment curieux à examiner.

Dans le centre du fuseau les fils sont fortement rallongés. Dans certains cas ils sont tellement digérés que la matière achromatique coule, elle difflue complètement; les fils se déforment. On a donc bien sous les yeux la même matière achromatique ramenée à cet état fluide, visqueux, qu'elle avait au moment où ses particules, disséminées, éparses dans le caryoplasma, respirant enfin d'une façon suffisante, ont été entraìnées à la suite des bâtonnets en mouvement et cherchant eux-mêmes à respirer. C'est donc bien la reproduction exacte d'un état antérieur qu'elle retrouve et laisse constater à l'observation, en répétant, point par point, cette série de réactions, par lesquelles elle a passé pour former les fils.

La phase que je viens de décrire est excessivement courte, plus courte que celle que j'ai montrée précédemment, et pendant laquelle on peut voir les fils dans le noyau encore fermé par la membrane nucléaire. Il est évident que ces périodes n’ont pas été constatées, tout simplement parce que ce n'est qu'à la suite de recherches très longues, très pénibles, que l'on arrive à trouver quelques coupes oủ l'on peut les voir. Ainsi parvient-on à s'expliquer comment de très bons observateurs ont pu conclure que la marche de la division devait s'effectuer dans le fuseau du noyau primaire du sac, comme dans les noyaux de l'albumen où il est beaucoup plus facile de trouver une grande quantité de noyaux, surtout dans la première couche endospermique, à toutes les phases de la division. Malheureusement les noyaux d'albumen sont beaucoup plus petits, les détails y restent imperceptibles.

La période qui va suivre est un peu plus longue. On la trouve plus souvent. Les bâtonnets commencent à se condenser. Au même moment toutes les matières interposées aux demi-plaques se condensent : chose étonnante, les fils se reforment; il n'y a pas à en douter; on les voit restaurés; ils redeviennent droits et ils se con- 
tractent au point de produire un phénomène extrêmement curieux et très important à constater.

Lorsque les demi-plaques vont enfin arriver aux pôles, qu'elles commencent à respirer mieux, qu'elles cessent d'empêcher de respirer, en leur faisant concurrence, les matières protoplasmiques, fils, etc., interposés entre elles, ces derniers respirent plus complètement, produisent des forces vives, se condensent, se contractent.

De l'autre côté des demi-plaques, entre elles et les pôles, les fils qui forment les pointes du fuseau, fils parfaitement distincts jusque-là et digérés seulement au contact des demi-bâtonnets, recondensés aussi vite qu'ils sont digérés, n'ont jamais cessé, à partir de leurs points anastomosés avec la pointe des demi-bâtonnets, de tirer sur cette pointe et par conséquent de faire progresser vers les pôles les demi-bâtonnets. 0r, quand les demi-plaques arrivent à une petite distance des pointes du fuseau, tous les fils, même ceux qui en divergeant dans le cytoplasma empêchaient le rapprochement trop rapide des deux pointes du fuseau, en faisant le parachute, tous sont subitement hydratés, digérés, ramollis, et nécessairement sans qu'on puisse cette fois en douter, par le voisinage des demi-plaques, qui arrivent enfin. Alors les fils cassés, raidis, tétanisés autour des pointes du fuseau, sont ramollis, assez pour plier, pour perdre leur rigidité; ils fléchissent, se recourbent en cédant au tirage des fils centraux du fuseau, qui, eux, se contractent et deviennent rigides, se raidissent à leur tour, au même moment. Ainsi les pointes du fuseau composées : $1^{\circ}$ des fils cassés, raidis, tétanisés sur toute leur longueur, formant parachute; $2^{\circ}$ des extrémités des fils continus d'un pôle à l'autre dans le fuseau et qui vont tirer sur les fils tétanisés; $3^{\circ}$ des fils qui tirent sur les pointes des demi-bâtonnets, ces trois catégories de fils sont toutes ramollies; toutes subissent l'influence des demi-plaques, de la nuclèine, comme toutes les matières interposées entre elles l’ont précédemment subie. La nucléine en approchant des pointes du fuseau répète sur elles ce qu'elle a fait sur les parties centrales du même fuseau. Alors ces pointes du fuseau fléchissent, s'aplatissent et rentrent dans le centre des noyaux filles. On voit distinctement tous les fils cassés, raidis tout à l'heure sur toute leur longueur jusqu'aux pointes, recourbés maintenant à leur extrémité polaire ramollie, plonger dans les noyaux filles. Its sont cependant 
encore raidis, mais à une certaine distance de ces derniers, autour desquels ils se redressent progressivement; de sorte que, lorsqu'on ne les a pas suivis pas à pas, on serait tenté de les prendre pour des irradiations polaires; tandis que ce ne sont que les fils superficiels du fuseau cassés par zones successives.

L'affaissement des pointes du fuseau, facile à constater sur le fuseau du Lis blanc, à cause de ses grandes dimensions, prouve jusqu'à l'évidence que ce sont bien les demi-plaques qui produisent les phénomènes d'hydratation, qui ramollissent, digèrent tous les fils, tout le fuseau, puisque, à leur approche, les pointes du fuseau, rigides, résistantes jusque-là, se ramollissent à leur tour.

Sur des préparations avantageuses, lorsque l'un des noyaux filles, comme on le voit dans une de mes préparations, se présente non point de face, mais légèrement de còté, en l'examinant avec un objectif à immersion homogène, on voit à merveille les pointes des demi-bâtonnets, par conséquent les parties médianes des demibâtonnets, recourbées en dedans des noyaux filles restaurés. Ces pointes forment un (C) bien visible. Les extrémités de chaque demi-bâtonnet se soudent aux extrémités voisines du demi-bâtonnet contigu. Le filament se reconstitue, se contracte et fait sortir une certaine quantité de matière achromatique qui n'a pas pu s'écouler le long des fils.

Avec l'affaissement des pointes du fuseau, le fait dominant, c'est celui des fils centraux digérés au point de s'allonger, et que l'on voit ensuite devenir droits et se contracter. La matière achromatique dont ils sont formés est redevenue molle, fluide. Puis, aussitôt qu'elle a pu respirer suffisamment, qu'elle a pu produire des oxydations suffisantes, elle s'est condensée, a acquis de la cohésion, et s'est contractée.

Autour de la zone centrale du fuseau la digestion des fils a été moins complète. La matière achromatique ramollie, rendue plụs perméable, a produit plus vite de l'énergie et s'est contractée aussitôt, de sorte que les effets de la digestion ne sont apparents que sur les fils centraux; sur tous ceux qui respirent comme le protoplasma cellulaire voisin du fuseau, on n'a pas le temps de les voir. Il en est de même pour les fils situés entre les demi-bâtonnets et les pôles. Les demi-bâtonnets digèrent légèrement, et sur une faible longueur, les fils en rapport avec eux, en produisant une petite couche unissante, une petite anastomose qui les fait adhérer 
aux fils contigus. La surface du demi-bâtonnet en contact avec eux se ramollit aussi légèrement, mais la respiration est plus complète sur la pointe du demi-bâtonnet; aussitôt ramollis, fils, couche unissante, demi-bâtonnet respirent, produisent de l'énergie, se contractent, et les fils font avancer le demi-bâtonnet. La condensation de celui-ci n'est que superficielle; les couches de linine situées plus profondément ne se condensent pas. Il en est de même pour les fils situés entre les demi-plaques. Les parties centrales respirent moins, restent plus longtemps digérées, pendant que les effets des digestions sont supprimés à mesure qu'ils se produisent sur les fils qui respirent comme le cytoplasma contigu au fuseau.

Les demi-bâtonnets sont donc attirés vers les pôles par des fils auxquels ils adhèrent, et qui se contractent parce qu'ils sont ramollis et respirent mieux. Placés, en second lieu, au milieu de matières très oxydables qui leur font une concurrence avantageuse puisqu'elles sont toujours situées plus superficiellement, ils conservent, surtout dans leurs parties tournées vers la zone centrale, une plus grande diffluence qui permet à la nucléine de diffuser vers cette région, et c'est pour cela que celle-ci est toujours dans un état de digestion plus complète. La quantité de matières qui cherchent à respirer est trop grande pour la quantité d'oxygène qui peut y pénétrer.

La condensation des demi-bâtonnets, puis du filament reconstitué aux pôles, se fait en même temps que la condensation dans la zone équatoriale d'une certaine quantité de matière achromatique qui a été digérée dans les fils, au point de devenir tout à fait liquide. Lorsque les demi-bâtonnets ont été sur le point d'arriver aux pôles, on a pu remarquer qu'entre les deux demi-plaques la plupart des fils, en redevenant droits, en se contractant, étaient plus gros. Les auteurs ont pensé que ces fils, qu'ils ont appelés les fils unissants, parce qu'ils sont situés entre les demi-plaques, étaient des fils nouveaux. Ce sont des fils fins agglutinés, collés ensemble, des fils coalescents, comme ceux que M. Guignard a vus (Annales, $1885,1891)$ à l'extérieur du fuseau, et sur lesquels je donnerai quelques détails tout à l'heure.

Les fils unissants sont donc des fils fins collés ensemble, et collés par la matière achromatique elle-même des fils qui a été partiellement ramenée à l'état liquide. Dans cet état, étant très mobile, 
elle a en partie diffusé entre les fils, mais une autre partie, en se condensant, s'est attachée à eux, elle s'est mise à les suivre pour se rendre dans leur partie centrale, en s'éloignant des demi-plaques oủ la respiration est réduite. Quand la respiration a commencé à augmenter dans la région équatoriale, la matière attachée aux fils s'est avancée dans cette région pour y respirer davantage. Elle ne possédait pas assez de cohésion, dans la région voisine des demiplaques. Elle était bien attachée aux fils, mais pas assez pour les coller complètement; elle a donc pu glisser sur eux et, en acquérant de la cohésion, coller les fils fins dans la région équatoriale. Les fils fins qui forment les fils unissants en sont alors débarrassés dans la région voisine des demi-noyaux. On voit ces fils fins, mais seulement dans les régions voisines des demi-noyaux; sur toute leur partie médiane ils sont encore collés, et forment les gros fils de la région équatoriale : le rudiment de plaque cellulaire.

M.Guignard a vu les fils fins débarrassés de la substance achromatique qui les collait, et il les a dessinés. En regardant les figures qu'il a données de la reconstitution des demi-plaques ou noyaux filles (Annales, 1885, 1891), on peut se convaincre qu'il a dessiné des fils plus nombreux dans les parties voisines des demi-noyaux. Mais il n'a pas remarqué que la substance collante qui les unit, et qui n'est autre chose que de la matière achromatique devenue plus colorable parce qu'elle s'est chargée d'une certaine quantité de nuclèine diffusée alors plus abondamment à partir des noyaux filles par les demi-bâtonnets plus diffluents, que cette matière achromatique quitte les fils fins vers leurs parties les plus rapprochées des noyaux filles et s'avance ensuite vers leur région médiane, c'est-à-dire dans la zone équatoriale, afin d'y respirer plus complètement.

Nous allons voir la même substance collante produire les fils coalescents extérieurs du fuseau que M. Guignard a encore décrits en 1891 (Annales).

Pour arriver à se faire une idée exacte du fuseau, il faut l'ouvrir par une coupe longitud tnale et axiale, après l'avoir imbibé soigneusement de paraffine. Quand on réussit à obtenir une coupe convenable dans laquelle les fils intérieurs qui sont extrêmement délicats n'ont pas été dérangés par le rasoir, on voit, non sans étonnement, que dans l'intérieur du fuseau il existe une multitude de fils continus d'un pôle à l'autre, et qui ne sont pas droits. 
Ces fils sont flexueux et semblent s'anastomoser par places les uns aux autres.

M. Guignard a vu ces fils, qui sont très fins, entre les gros fils coalescents dont je vais parler dans un instant. Dans son Mémoire de 1891, il a formellement dit qu'il existait, à côté des fils coalescents, des fils plus fins, et que les premiers étaient tout simplement formés par la réunion de plusieurs fils fins. Mais il n'a pas pu se rendre compte que ces fils fins intérieurs ne sont pas droits; pour le voir, il faut ouvrir le fuseau.

Pour bien voir ce que sont les fils coalescents il, faut aussi ouvrir un fuseau par le milieu et longitudinalement, lorsque les bâtonnets sont en train de se partager. Dans les coupes fixées à l'alcool absolu, comme je l'ai indiqué, on trouve certains détails intéressants, qu'il n'est pas possible de voir quand on a employé, même abondamment, l'alcool rectifié, ou l'alcool absolu en quantité insuffisante.

Les bâtonnets, en finissant momentanément leurs mouvements dans la partie médiane du fuseau, cherchent à s'approcher des parois du sac embryonnaire: car, eux aussi, ont besoin de respirer. Par leur extrémité la plus rapprochée du centre du fuseau, ils se partagent en deux tronçons. Ils ont préalablement, pendant leur course à travers le caryoplasma, augmenté leur activité; mais la nucléine n'a pu hydrater, ramollir qu'une tranche médiane dans leur substance. C'est dans ces conditions qu'ils viennent se ranger dans la plaque nucléaire; et c'est seulement sur leur extrémité la moins rapprochée du cytoplasma que s'opère le décollement des deux tronçons formés. Dans cette partie, le bâtonnet respire moins; il est plus actif, il digère légèrement les fils en contact et les matières protoplasmiques roisinez de son extrémité. Aussitòt les matières digérées s'attachent aux fils, et en les suivant cherchent à gagner des points où elles peuvent mieux respirer qu'au voisinage des bâtonnets qui leur font concurrence. Mais, très rapidement, les fils ramollis et les matières qui s'y sont collées respirent et se contractent. Les fils accolés diminuent de longueur. En un temps très court, les fils, digérés sur une partie de leur longueur, ont respiré très vite et produit la même quantité d'énergie qu'ils avaient produite depuis leur naissance; ils ont produit une contraction plus considérable, alors le fil coalescent en formation se raccourcit plus vite que les fils fins du fuseau. 
Chaque fil coalescent en formation se raccourcit, tire sur les pôles et en mème temps sur les tronçons de bâtonnet. Les autres portions de bàtonnet viennent tour à tour se présenter au niveau du fil coalescent, en produisant une nouvelle digestion supprimée aussitôt que produite, et suivie d'une production de matière coalescente. Quant à l'extrémité du bâtonnet la plus rapprochée des couches voisines du caryoplasma, elle respire plus que la partie qui vient se présenter et se décoller dans l'alignement du fil coalescent; elle ne produit ni hydratation, ni matière coalescente. Les fils qui sont en rapport avec elle ne se collent pas; ils forment un réseau à mailles bien distinctes autour d'elle.

On peut ainsi arriver à se faire une idée de l'utilité des fils fins préparés dans le noyau; non seulement ils fournissent aux bâtonnets pendant leur marche une matière protoplasmique de digestion facile, capable de se contracter en respirant, mais ces fils nombreux, en contact avec les diverses parties des bâtonnets, servent de fils conducteurs, d'appareil collectionneur destiné à arrèter la matière achromatique qui est digérée, remise en œuvre par la nucléine après la disparition de la membrane nucléaire, quand la diffusion de l'oxygène se fait mieux dans les parties centrales de cette masse énorme de protoplasma qui emplit le sac embryonnaire du Lis blanc. Les matières protoplasmiques du noyau, remaniées pendant leur course par les bâtonnets, respirent mieux, acquièrent de la cohésion, s'attachent en partie aux fils formés et les épaississent, en les fortifiant progressivement. Il en est de même quand le fuseau est formé. Les fils fins qui sont en rapport avec les diverses parties en réaction des bâtonnets reçoivent et collectionnent une partie des matières digérées qui se condensent à leur voisinage. Celles-ci se fixent à leur surface, aussitôt produites, respirent, produisent des forces vives qu'elles cèdent aux fils oủ elles se sont collées, en augmentant la quantité d'énergie qui peut être produite sur une longueur déterminée.

Les fils coalescents font faire aux demi-bâtonnets étendus contre le cytoplasma une partie du chemin qu'ils doivent accomplir vers les pôles. L'activité des demi-bâtonnets n'est atténuée qu'un instant, et seulement sur leur surface externe. Comme on l'a vu plus haut, leur surface interne devient bientôt assez diffluente pour que les deux parties du demi-bâtonnet, plié en deux, puissent se coller et se confondre. Le centre d'activitẻ de chaque demi-bàton- 
net se replace sur le milieu de leur longueur, pendant que le fuseau commence à s'élargir et en même temps à se raccourcir. Avec l'élargissement du fuseau les demi-bâtonnets sont ramenés progressivement dans une direction perpendiculaire aux fils du fuseau; puis on voit qu'ils sont tirés davantage par les fils qui sont en contact avec leur partie médiane. Celle-ci agit plus complètement sur eux et les fait contracter plus vite vers les pỏles.

Entre les moitiés de plaque qui s'éloignent, pendant que le fuseau s'élargit et se raccourcit, une partie des fils s'accolent et forment les fils unissants, entre lesquels on voit des fils moins épais. A l'extérieur les fils se rompent et continuent à diverger, en se raidissant, dans le cytoplasma.

On arrive ainsi à cette phase pendant laquelle, les bâton nets ramollissant tout le fuseau, il est possible de constater à la simple observation les effets des digestions accomplies plus complètement sur ses parties centrales, en attendant que les parties externes qui tirent les demi-plaques vers les pôles, en participant elles-mêmes, à leur tour, aux réactions qui se sont réalisées sur les fils unissants, se ramollissent à leur tour et s'affaissent. Alors on trouve la preuve formelle indiscutable que ce sont bien les moitiés de plaque qui en s'approchant des pointes du fuseau produisent sur ces pointes, comme sur les parties centrales, dont elles se sont éloignées, les phénomènes d'hydratation qui ont amené la digestion du fuseau.

Les bâtonnets ne peuvent se diviser sans digérer une tranche médiane de leur linine. Les deux tronçons formés ne peuvent être entraînés vers les pôles qu'autant que les fils qui subsistent entre eux ne se contracteront pas en même temps que ceux qui les tirent vers les pôles. Les demi-bâtonnets sont donc attirés d'un côté par des fils qui se contractent, et ils glissent au milieu d'autres fils qui se raidissent, en les assujettissant dans leur marche.

Considérée dans son ensemble, chaque demi-plaque se trouve sur la limite d'une matière protoplasmique contractile au milieu de laquelle elle s'introduit en vivant, en respirant et en assimilant; mais en vivant d'une vie restreinte, comme la respiration à laquelle elle est fatalement condamnée, au milieu de matières qu'elle crée, semble-t-il, dans le seul but de se faire faire une concurrence incessante; de manière, en respirant moins, à ménager l'énergie propre qu'elle possède, qu'elle dépense en réactions. De sorte que 
les matières qui l'environnent, en brûlant, tendent à devenir inertes, en acquérant de la cohésion, tandis qu'elle tient en réserve le moyen de les rendre à la vie, à l'activité, en leur faisant perdre cette cohésion : en les ramollissiant, en les liquéfiant.

Chaque demi-plaque marche, pourrait-on dire, comme le tube pollinique au milieu du tissu conducteur du style, en vivant aux dépens des matériaux qui l'entourent. Sur sa partie externe les effets progressifs des digestions qu'accomplit la demi-plaque sont supprimés aussi vite qu'ils sont produits; sur la partie interne, elle respire moins, reste plus diffluente, en même temps que toutes les matières en contact. La distance entre les deux demi-plaques n'augmente qu'insensiblement. L'action combinée qu'elles exercent ainsi sur les parties interposées entre elles, aussitôt leur séparation, se prolonge, grâce au tirage qu'exercent, à la périphérie, les fils qui respirent comme le cytoplasma voisin du fuseau et qui, de part et d'autre, sont attachés aux pôles. Les pôles sont donc amenés vers les demi-plaques autant que celles-ci marchent vers eux. Le fuseau, grâce à ce mécanisme merveilleux, vient au-devant des demi-plaques auxquelles il fournit, non seulement les matériaux nécessaires à leur marche, à l'aide des adhérences qui se produisent entre les fils et les demi-bâtonnets, mais il fournit aussi aux demi-plaques une somme d'énergie qu'elles emploient en réactions de plus en plus actives, jusqu'au moment où elles arrivent en deux points oủ leur activité est atténuée et suspendue. Alors le fuseau a accompli sa destinée, il s'affaisse et rentre en partie dans le noyau pour servir d'aliment facile à de nouvelles réactions. Dans la progression des demi-plaques, le fuseau a été secondé par des causes auxiliaires qui sont elles-mêmes des effets secondaires et naturels de l'activité de celles-ci. La turgescence du tonneau, produite à un moment donné entre elles, a contribué certainement par la pression exercée aussi bien sur les faces inférieures, internes, des demi-bâtonnets, que sur les parties latérales du fuseau, à faire progresser les demi-plaques vers deux points où elles sont appelées aussi pour respirer plus complètement qu'elles ne peuvent le faire du côtté des surfaces qu'elles ont précédemment décollées. Entre ces surfaces décollées il s'est formé, dès la séparation des demi-plaques, un espace où la respiration esı forcément réduite, puisque la surface décollée de chaque demibâtonnet, la surface interne de chaque demi-plaque, respire à ses 
dépens, en faisant concurrence aux fils et aux matières interposées qui s'y trouvent soumises, dès lors, à peu près aux mêmes conditions de respiration restreinte que celles qui existaient dans le noyau avant la disparition de la membrane nucléaire. On se rendra compte de cette circonstance, en se rappelant que les demi-bâtonnets, dans le fuseau, ont augmenté leur activité au milieu de matières qui s'oxydent autour d'eux, et qui les empêchent eux-mêmes de s'oxyder, de respirer, au moment où ils auraient besoin d'absorber plus d'oxygène qu'ils ne pouvaient le faire dans le noyau quand leur activité ne faisait que commencer à croître. Entre les demi-plaques, la production de forces vives, sans y être complètement suspendue, s'y trouve diminuée, au point que toute condensation, et surtout que toute contraction ne peut s'y produire. C'est d'ailleurs une condition sans laquelle les demiplaques ne pourraient se séparer.

En jetant un coup d'œil d'ensemble sur les faits acquis pendant le cours de cette étude, on remarquera que, chez les Spirogyra comme chez le Lis blanc, on est forcément amené à envisager le rôle rempli par le noyau en division, d'une façon différente de celle qui a été acceptée.

Le noyau prépare une matière destinée à respirer et, en respirant, à produire des forces vives. Il la prépare derrière sa membrane; puis, lorsqu'elle est à point, il fait intervenir la respiration cellulaire, en rendant la membrane nucléaire plus perméable.

Chez le Lis blanc au milieu de la masse énorme de protoplasma qui emplit le sac embryonnaire, la respiration, dans les parties centrales, est plus réduite que chez les Spirogyra oủ le noyau est pour ainsi dire en rapport immédiat avec le milieu extérieur. Aussi, chez le Lis, le caryoplasma modifié respire lentement : les phénomènes merreilleux qui se passent dans le fuseau et qui sont si différents, à des distances pourtant infinitẻsimales, sur des fils distants les uns des autres de quelques millièmes de millimètre, le montrent suffisamment. Lorsque la. membrane nucléaire, modifiée comme nous l'avons vu, met le protoplasma nucléaire en rapport plus complet avec l'extérieur, on voit les fils du fuseau, fils rudimentaires, sans cohésion, apparaitre dans le noyau. Le noyau a trouvé enfin la source de forces vives nécessaires au travail qu'il va accomplir, qu'il accomplit sous les yeux de l'observateur. Il forme immédiatement, dans son caryoplasma, 
plus largement alimenté par le sac cellulaire; respirant mieux, des corps fortement oxydés : de l'eau, de l'acide carbonique, etc., soustraits à d'autres molécules qui se chargent d'énergie, à poids moléculaire élevé, d'une extrême instabilité, qui se condensent; éparses d'abord, disséminées, elles se réunissent, sont entraînées par les bâtonnets en mouvement, forment des traînées, puis des fils, c'est-à-dire un tissu progressivement amené à cet état de perfection que nous lui avons vu dans le fuseau.

Dans le fuseau, ce tissu, agencé d'une façon admirable, obéit aux mèmes réactions; il produit, là où il le faut, des forces vives, et se contracte subitement; ailleurs il se laisse ramollir et permet aux parties qui doivent se séparer de pouvoir le faire; et tout cela, parce que ce tissu, dans le fuseau comme dans le noyau, à la fin de la courte vie qu'il a, comme au moment de sa naissance, est mis en œuvre, se trouve sous la dépendance d'une cause qui agit au milieu de lui, qui le remet dans de bonnes conditions de respiration.

Dans le sac embryonnaire du Lis, les matières créées par le noyau respirent lentement, s'organisent lentement, pendant toute une longue période, avant de trouver la cohésion sans laquelle toute contraction serait impossible. Et, quand elles ont trouvé enfin cette cohésion, ce sont de longs fils qui se raccourcissent de moitié qui font la besogne à faire. Elle consiste, après une énorme contraction, à faire séparer les deux moitiés de noyau, de manière qu'elles ne se réunissent pas. Ici l'instrument destiné à aider à cette séparation a des dimensions en rapport avec la faible respiration que possède la région centrale du cytoplasma. La respiration est réduite; les forces vives nécessaires le seront aussi. Il faut une grande quantité de matière à brûler; le fuseau est énorme.

Quand on veut bien partir de cette considération, ce qui se passe ailleurs, aussi bien que chez les Spirogyra, on peut le dire sans crainte de généraliser, devient compréhensible.

Dans toute cellule oủ la respiration se fait mieux qu'au centre du sac embryonnaire du Lis blanc, les forces vives nécessaires à la séparation des chromosomes seront plus faciles à obtenir; la matière achromatique, la partie achromatique du noyau en division, sera réduite, elle devra quelquefois, sous un petit volume, 
avec des fils achromatiques imperceptibles, produire l'effet nécessaire.

Chez les Spirogyra, par exemple, les rapports différents qui existent entre la cellule, le noyau et le milieu extérieur rendent même nécessaire l'expulsion préalable d'une partie de la matière achromatique en dehors du noyau, avant que, la membrane étant modifiée, l'oxygène puisse pénétrer jusqu'au filament d'une façon assez abondante pour faire recondenser la linine et arrêter au sein de celle-ci toute réaction de la nucléine. Alors seulement qu'il a accompli la moitié de sa tache, le filament travaille à faire disparaître la membrane, en préparant la seconde partie de la matière achromatique; alors on voit les deux parties de celle-ci s'anastomoser par la disparition de la membrane ou plutôt des parties de membrane qui les séparaient, et toute la matière achromatique, tous les fils se contracter énergiquement entre les cordons suspenseurs, en emprisonnant dans leur partie médiane le filament qui a conservé l'activité nécessaire pour se diviser. Et en effet, après un court repos, il ramollit la partie médiane de ses anses tassées dans la plaque nucléaire; il agit en même temps sur les fils, les remet dans de bonnes conditions de respiration, et les fils agissent comme chez le Lis. Les demi-plaques qui s'empêchent mutuellement de respirer s'éloignent l'une de l'autre, en produisant un tonneau qui aide aussi à leur séparation, en protégeant les parties internes des demi-noyaux.

Comment peut-on concevoir que la nucléine peut hydrater, digérer les matières protoplasmiques qui l'environnent? En produisant des corps hydrogénés instables, dont l'hydrogène mis en liberté, à l'état naissant, très diffusible, provoque, en l'absence de l'oxygène libre, les réactions très connues par hydratation qui se réalisent dans toutes les digestions des matières protoplasmiques : grasses, hydrocarbonées ou azotées.

Dans la nucléine assujettie pendant son existence à une respiration réduite, la présence de corps instables chargés d'énergie, remplissant le rôle de ferments puissants, est possible. C'est quand la nucléine respire le moins, qu'elle est le plus active. C'est lorsqu'elle va être amenée à l'état de vie sans air, qu'elle va être totalement privée d'oxygène libre, qu'elle acquiert sa puissance d'action maximum. Ainsi est-on amené à constater que la nucléine, que le filament qui la contient, tend pendant certaines périodes de 
son existence à devenir un être anaérobie, remplissant les fonctions visibles, constatables à l'observation, que nous lui avons vu remplir; et les remplissant avec le plus de puissance lorsqu'il va être privé d'air d'oxygène libre, et qu'il est obligé de vivre aux dépens de l'oxygène combiné des matières qui l'environnent.

SUPPLÉMENT A LA LISTE DES PLANTES RARES OU INTÉRESSANTES (PHANÉR0gaMES, CRYPTOGAMES SUPÉRIEURES ET CHARACÉES) DES ENVIRONS DE MONTFORT-L'AMAURY ET DE LA FORET DE RAMBOUILLET (SEINE-ET-OISE); par M" Marguerite BELEZE (1).

Myosurus minimus L. - Champs humides. Plaine de $\mathbf{M}^{\mathrm{t}}$ et à SaintLéger (F. de R.).

Ranunculus hederaceus L. - Rigoles, dans la cour de la ferme Larcher (Saint-Léger); F. de R.

R. Delacouri G. et Mab. - Bois humides. Allée de Saint-Laurent, routes des Longues-Mares et de Vitry (F. de R.).

R. Questieri Billot. - Mêmes localités et poteau du Chêne-Montavale (F. de R.).

Helleborus fotidus L. - Bois de Beynes et vieux murs à Galluis, près $\mathbf{M}^{\mathrm{t}}$.

Diplotaxis muralis DC. - Champs, à Méré, près $\mathrm{M}^{\mathrm{t}}$, où il tend à disparaître.

Teesdalia nudicaulis R. Br. - Pelouses sèches. Autour du Dolmen dit de la "Pierre-Ardroue » et au croisement de la route aux Vaches avec celle de Bazôches (F. de R.).

Helianthemum guttatum Mill. - Sables chauds et arides du chemin de la «Pierre-Ardroue " au Jardinet (Saint-Léger), et berges de l'étang du Roi (Poigny); F. de R.

Viola canina L. - Lieux arides, aux mares Moussues, plaine de $\mathbf{M}^{\mathbf{t}}$; routes des Fonds aux Mesnuls, de Saint-Léger à Montfort, près le parc d'En-Haut et carrefour du Monnereau (F. de R.).

Parnassia palustris L. - Prairies spongieuses et tourbeuses. Prés Jaunot, à Gambayseuil et au Maupas (Saint-Léger); F. de R.

Polygala depressa Wender. - Bruyères, et parmi les Ajoncs; plaine

(1) Voy. le Bulletin, t. XLII, p. 49 (1895). Comme précédemment, M' et $F$. de R. sont les abréviations de Montfort-l'Amaury et de Forêt de Rambouillet. 


\section{$2 \mathrm{BHL}$ Biodiversity Heritage Library}

Degagny, Charles. 1896. "Recherches Sur La Division Du Noyau Cellulaire Chez Les Végétaux ( $8^{\circ}$ Note)." Bulletin de la Société botanique de France 43, 332-346. https://doi.org/10.1080/00378941.1896.10830684.

View This Item Online: https://www.biodiversitylibrary.org/item/8665

DOI: https://doi.org/10.1080/00378941.1896.10830684

Permalink: https://www.biodiversitylibrary.org/partpdf/160052

\section{Holding Institution}

Missouri Botanical Garden, Peter H. Raven Library

\section{Sponsored by}

Missouri Botanical Garden

\section{Copyright \& Reuse}

Copyright Status: Public domain. The BHL considers that this work is no longer under copyright protection.

This document was created from content at the Biodiversity Heritage Library, the world's largest open access digital library for biodiversity literature and archives. Visit BHL at https://www.biodiversitylibrary.org. 